
\title{
$\begin{array}{ll}\text { Research Square } & \text { Preprints are preliminary reports that have not undergone peer review. } \\ \text { They should not be considered conclusive, used to inform clinical practice, } \\ \text { or referenced by the media as validated information. }\end{array}$
}

\section{Effect of Lopinavir/ritonavir Treatment on COVID-2019 Transmissibility: a Possible Option to Reduce Isolation Time}

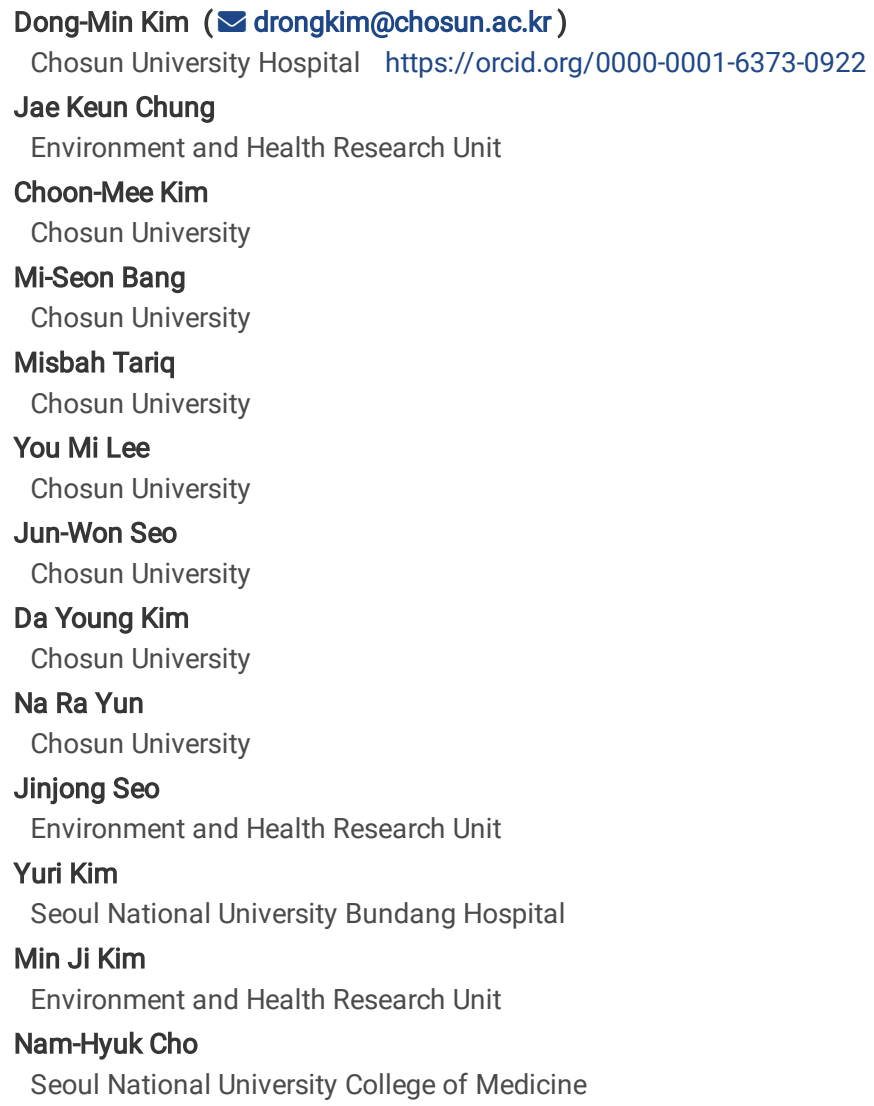

Research Article

Keywords: COVID-19, viable, SARS-CoV-2, Lopinavir/ritonavir, cell culture

Posted Date: May 24th, 2021

DOI: https://doi.org/10.21203/rs.3.rs-516757/v1

License: (1) This work is licensed under a Creative Commons Attribution 4.0 International License. Read Full License 


\section{Abstract}

Background: Owing to the coronavirus disease 2019 (COVID-19) pandemic, there is a shortage of hospital wards to accommodate the increasing number of patients, especially in intensive care units. Healthcare systems are collapsing in many countries. Therefore, it is necessary to reduce isolation time.

Methods: We examined the effect of lopinavir/ritonavir administration in patients with SARS-CoV-2. To assess the viral load, duration and clearance of viable virus; cell culture and RT-PCR were performed in parallel.

Results: No viable SARS-CoV-2 could be detected after administration of lopinavir/ritonavir with median time of viable viral clearance being one day after administration. The mean viral load in both upper and lower respiratory tract samples of lopinavir/ritonavir administered group was significantly lower than the group who were not treated with any antiviral agent. The duration of viable viral shedding was shorter in patients with lopinavir/ritonavir treatment compared with those without treatment.

Conclusion: This study suggests that lopinavir/ritonavir treatment offers a possible method to reduce isolation time of patients infected with the SARS-CoV-2.

\section{Background}

The Coronavirus Study Group of the International Committee on Taxonomy of Viruses designated the novel coronavirus 19 as the severe acute respiratory syndrome-coronavirus-2 (SARS-CoV-2). ${ }^{1}$ Some variants, including the G614 polymorphism, B.1.1.7 variant, and B.1.351 lineage, have much higher transmissibility than others. ${ }^{2}$ Due to this pandemic, there is a shortage of hospital wards, especially in intensive care units, to accommodate the increasing number of patients; healthcare systems have been collapsing in many countries.

Non-test-based and test-based strategies are used to determine the duration for which patients with COVID-19 must take the necessary precautions and isolate themselves. ${ }^{3,4}$ The test-based strategy asserts that individuals may discontinue isolation if they test negative in two successive SARS-CoV-2 molecular viral assays (using respiratory specimens) performed at least $24 \mathrm{~h}$ apart. However, the greater the age of the patient and the severity of infection, the greater the viral RNA shedding. This viral RNA can, on average, be detected for up to 18 days after symptom onset and in some cases, it may be detected in the respiratory tract several months after a person is infected. ${ }^{5}$ Since detectable viral RNA does not indicate an infectious virus, the test-based strategy may not effectively reduce the duration of the isolation period.

SARS-CoV-2 has the highest transmissibility in the early period of infection, immediately before the onset of symptoms. A Taiwanese study reported that secondary infection usually occurs up to 5 days after symptom onset. ${ }^{6}$ Based on these findings, the Center for Disease Control and Prevention (CDC) proposed a non-test-based strategy in which mild to moderate COVID-19 patients may discontinue infection control precautions if their temperature is normal for $24 \mathrm{~h}$ without the use of antipyretics and their symptoms, such as cough and shortness of breath, abate after at least 10 days from onset. ${ }^{3}$

However, there are insufficient data to determine whether all patients with mild to moderate COVID-19 require 10 days of precautions and isolation after symptom onset and whether isolation may be discontinued earlier. No study has investigated the duration of viral shedding in immunocompetent COVID-19 patients who have taken antiviral agents such as lopinavir-ritonavir (Kaletra). Therefore, the present prospective observational study aimed to investigate this relationship between the shedding of the virus cultured from patients administered lopinavir/ritonavir.

\section{Methods}

Seven patients were admitted to a government-approved, nationally designated quarantine ward in Chosun University Hospital between February 2020 and April 2020 and were tested using PCR, viral culture, and immunofluorescence assay (IFA).

The patients were confirmed to be positive using real-time reverse transcription-polymerase chain reaction (RT-PCR) targeting at least two or more genes or who showed $>$ four-fold increase or seroconversion in the antibody titers against SARS-CoV-2.

\section{Sampling and RNA extraction}

Nasopharyngeal and oropharynx swabs and sputum samples were collected using commercial UTM ${ }^{\text {TM }}$ kits containing $1 \mathrm{~mL}$ of viral transport media (Noble Bio, Korea) and used for RNA extraction. The viral RNA was extracted using a fully automated instrument (PCL South Korea) and Real-prep viral DNA/RNA Kit (Biosewoom, South Korea). RT-PCR was performed to target the NP, E, and RdRp genes, along with a positive reference gene, using distilled water as a negative control. For the NP gene, the primers and probes were designed in-house, whereas for the E and RdRp target genes, Kogene kit (Kogene Biotech Seoul, South Korea) was used; manufacturers' specifications were used for the reaction conditions and amplifications.

\section{Cell culture}

The African green monkey kidney Vero E6 cell line was purchased from the Korean Cell Line Bank (KCLB no. 21587) and maintained in Dulbecco's modified Eagle's medium (DMEM, Gibco, Thermo Fisher Scientific, USA) supplemented with 10\% fetal bovine serum (FBS, Gibco, Thermo Fisher Scientific, USA) and 1X penicillin/streptomycin ( $\mathrm{PC} / \mathrm{SM})$ at $37^{\circ} \mathrm{C}$ in a humidified atmosphere of $5 \% \mathrm{CO}_{2}$.

\section{Virus isolation from clinical specimens}

To isolate SARS-CoV-2 from COVID-19 clinical specimens, samples were prepared by mixing $500 \mu \mathrm{L}$ of sputum with $200 \mu \mathrm{L}$ of $100 x$ penicillin/streptomycin $(\mathrm{PC} / \mathrm{SM})$ and then incubated at $4{ }^{\circ} \mathrm{C}$ with a vortex interval of $15 \mathrm{~min}$. After $1 \mathrm{~h}$, the samples were centrifuged at $1,600 \times \mathrm{g}$ for $20 \mathrm{~min}$, and the supernatant was 
collected, to be added to Vero E6 cells.

Vero E6 cells were cultured in 24-well cell culture plates at a density of $2.5 \times 10^{5}$ cells per well for $24 \mathrm{~h}$. The cells were washed with sterile phosphate-buffered saline.

Subsequently, $200 \mu \mathrm{L}$ of the collected supernatant was added to Vero E6 cells in $500 \mu \mathrm{L}$ DMEM with $2 \%$ FBS and $1 \mathrm{X}$ PC/SM and incubated at $37{ }^{\circ} \mathrm{C}$ for $3-5$ days.

The infected Vero E6 cells were checked daily for cytopathic effects (CPE) following the procedures used to detect SARS-CoV and MERS-CoV in previous studies. ${ }^{20,21} \mathrm{CPE}$ was identified when cell rounding, or detachment was observed under a microscope. The cells were scraped from the wells, and $200 \mu \mathrm{L}$ of cell suspension was transferred to a new 24-well plate with Vero E6 cells in $500 \mu \mathrm{L}$ medium and incubated at $37^{\circ} \mathrm{C}$ for $3-5$ days (passage 1 ). According to the protocol described above, the virus isolate underwent two passages. After two passages at intervals of 3-5 days, real-time RT-PCR was performed regardless of the presence or absence of CPE for further quantification of the viral RNA in the scraped cells.

All cell culture infection experiments were performed in a biosafety level-3 laboratory at the Health and Environment Research Institute of Gwangju City.

The whole-genome sequence of SARS-CoV-2 isolates was sequenced using a MiSeq next-generation sequencer with 150PE (IIlumina, San Diego, CA, USA), and sequence assembly was performed using Bowtie v.1.1.2 and samtools-mpileup (samtools v.1.9). The whole-genome sequences of the cultured isolates were registered in the Global Initiative for Sharing All Influenza Data (GISAID).

\section{Viral RNA extraction and real-time RT-PCR}

The virus isolate was confirmed using real-time RT-PCR. Viral RNA was extracted from $200 \mu \mathrm{L}$ of the cell suspension using a viral DNA/RNA extraction kit (cat no. ZP02201, Taiwan) in an automated nucleic acid purification system (ZiXpress-32) according to the manufacturer's instructions. RNA was eluted in $100 \mathrm{LL}$ RNase-free water. SARS-CoV-2 was detected using the Bioneer Exicycler 96 Real-time PCR kit (Bioneer Inc., Korea).

\section{Indirect IFA}

To perform the indirect IFA, SARS-CoV-2 samples obtained from the Korea Centers for Disease Control and Prevention were used to infect Vero E6 cells. To prepare the SARS-CoV-2 antigen slide, cells infected for 3 days were cultured on Teflon-coated well slides overnight at $37{ }^{\circ} \mathrm{C}$ in a $5 \% \mathrm{CO}_{2}$ environment and fixed with $80 \%$ acetone the next day. The patient serum was diluted using a two-fold serial dilution from 1:16 and then reacted with SARS-CoV-2 antigens in a moist chamber for $30 \mathrm{~min}$ at $37^{\circ} \mathrm{C}$. After washing, the slides were further incubated with 1:400 diluted secondary antibody (fluorescein isothiocyanate-conjugated anti-human IgM and IgG; MP Biomedicals, OH, USA). The slides were examined using a fluorescence microscope (Olympus IX73, magnification: 400x) after dispensing the mounting solution (Vector Laboratories). An IgG antibody titer of $\geq 1: 32$ was established as the cut-off using IFA on clinical samples from 15 health-screened individuals.

\section{Results}

Seven patients were enrolled during the study period. Four patients had mild symptoms and did not require oxygen therapy, one had pneumonia and required oxygen therapy, and two had severe symptoms and required a mechanical ventilator during hospital admission. A total of 103 samples were tested using a real-time reverse transcription-polymerase chain reaction (RT-PCR) assay; 60 of these were cultured for SARS-CoV-2 infectiveness. The presence or absence of viable SARS-CoV-2 at various times from onset of symptoms and cycle threshold (Ct) values in all serial respiratory samples are shown in Fig. 1. SARS-CoV-2 was isolated from 21 of 60 cultured samples (35\%). The viable virus was observed only in samples with Ct values $\leq 32.95$ (Fig. 1), with a median Ct value of

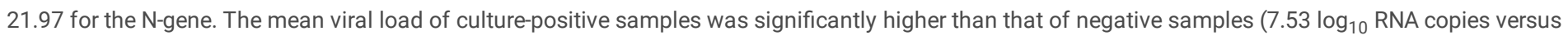
$5.64 \log _{10}$ RNA copies, $p=<0.0001$ ) (Figure S1).

To determine whether lopinavir/ritonavir inhibits SARS-CoV-2 replication, we compared the antiviral activity, viral viability, and clearance in a group of patients who received lopinavir/ritonavir $(n=4)$ with those of patients who did not $(n=3)$. A total of 47 samples from patients who were administered antiviral agents were tested via RT-PCR, and 15 were cultured for SARS-CoV-2. Viable viruses were found in only three of the 15 cultured samples (20\%) (Figure S1). Noticeably, no viable SARS-CoV-2 could be detected in immunocompetent severe COVID-19 patients administered lopinavir/ritonavir; the median time of viral clearance in cell culture was 1 day post-administration or hospitalization (Fig. 1b). For patients with mild symptoms who were not administered antiviral agents, 56 samples were tested via RT-PCR, and 46 were cultured for SARS-CoV-2. The virus was isolated from 18 of the 45 cultured samples (40\%). SARS-CoV-2 was continuously cultivated for up to 8 days after symptom onset; the median time of viral clearance in cell culture was 7 days after symptom onset (Fig. 1c), indicating prolonged shedding of viable SARS-CoV-2 in the absence of lopinavir/ritonavir treatment.

Although some samples from the lopinavir/ritonavir-treated group showed high viral loads ( $6 \log _{10}$ RNA copies), viable SARS-CoV-2 could not be isolated in cell culture (Figure S1). Overall, the mean viral load in both upper (4.12 versus $5.24 \log _{10}$ RNA copies; $p=0.0006$ ) and lower respiratory tract $(5.74$ versus 6.59 $\log _{10}$ RNA copies; $p=0.0226$ ) of all RT-PCR tested samples of lopinavir/ritonavir administered group were significantly lower than the group who were not treated with any antiviral agent. In both groups, the mean viral loads with respect to the days post hospital admission are shown in Fig. 2. We further evaluated the regression coefficient for the viral load and the percentage of cell culture positivity and observed a significant correlation between viral load and cell culture positivity rate in patients who were not treated with any antiviral agent $(p=0.01)$. However, no significant correlation was observed for the lopinavir/ritonavir-treated group $(p=0.12)$ (Figure S2). 
Patients 1-3 were isolated after being identified through contact tracing. They showed upper respiratory infection symptoms without pneumonia and were isolated without being given any antiviral treatment. They underwent nasopharynx, oropharynx, and sputum culture tests. The virus was continuously detected in the samples up to 8 days after symptom onset or hospital admission (Table 1). 
Table 1

Clinical information and real-time PCR and culture results of patients with COVID-19.

\begin{tabular}{|c|c|c|c|c|c|c|c|c|c|c|c|c|c|c|c|c|}
\hline \multirow{4}{*}{$\begin{array}{l}\text { Age/sex } \\
\text { Underlying } \\
\text { diseases }\end{array}$} & \multirow{4}{*}{$\begin{array}{l}\text { Status } \\
\text { / } \\
\text { PSI score }\end{array}$} & \multirow[t]{4}{*}{ Date } & \multirow{4}{*}{$\begin{array}{l}\mathrm{HD} \\
(\mathrm{SD})\end{array}$} & \multirow{4}{*}{$\begin{array}{l}\text { Antiviral } \\
\text { Agent }\end{array}$} & \multicolumn{12}{|c|}{ Respiratory tract specimens } \\
\hline & & & & & \multicolumn{4}{|c|}{ Nasopharynx } & \multicolumn{4}{|c|}{ Oropharynx } & \multicolumn{4}{|c|}{ Sputum } \\
\hline & & & & & \multicolumn{3}{|l|}{ PCR } & \multirow[t]{2}{*}{ culture } & \multicolumn{3}{|l|}{ PCR } & \multirow[t]{2}{*}{ culture } & \multicolumn{3}{|l|}{ PCR } & \multirow[t]{2}{*}{ cult } \\
\hline & & & & & $\mathrm{N}$ & $E$ & $\mathrm{RdRP}$ & & $\mathrm{N}$ & $\mathrm{E}$ & RdRP & & $\mathrm{N}$ & E & $\mathrm{RdRP}$ & \\
\hline \multirow{20}{*}{$\begin{array}{l}\# 1 \\
30 / M \\
\text { HTN }\end{array}$} & \multirow{20}{*}{$\begin{array}{l}\text { No } \\
\text { pneumonia } \\
\text { (No 02 } \\
\text { requirement) } \\
\text { / } 30\end{array}$} & \multirow[t]{2}{*}{$2 / 20$} & \multirow{2}{*}{\multicolumn{2}{|c|}{$\begin{array}{l}\# 0 \\
(0)\end{array}$}} & \multirow[t]{2}{*}{18.8} & \multirow[t]{2}{*}{18.0} & 18.0 & + & & & & & 20.8 & 20.2 & 20.2 & - \\
\hline & & & & & & & & & & & & & & & & \\
\hline & & $2 / 21$ & $\# 1$ & & 17.9 & 17.7 & 17.9 & + & 36.5 & 34.4 & 34.6 & - & 25.8 & 25.8 & 26.3 & - \\
\hline & & & (1) & & & & & & & & & & & & & \\
\hline & & $2 / 22$ & \#2 & & & & & & & & & & 18.6 & 18.5 & 18.7 & + \\
\hline & & & $(2)$ & & & & & & & & & & & & & \\
\hline & & $2 / 23$ & \#3 & & & & & & & & & & 32.1 & 33.7 & 32.1 & - \\
\hline & & & (3) & & & & & & & & & & & & & \\
\hline & & $2 / 24$ & $\# 4$ & & 27.5 & 27.5 & 27.7 & - & 27.6 & & & & 30.5 & 30.6 & 30.5 & - \\
\hline & & & (4) & & & & & & & & & & & & & \\
\hline & & $2 / 25$ & \#5 & & 31.4 & 31.8 & 31.9 & + & & & & & & & & \\
\hline & & & (5) & & & & & & & & & & & & & \\
\hline & & $2 / 26$ & \#6 & & 26.5 & 25.4 & 25.6 & + & 36.5 & 33.7 & 34.3 & & & & & \\
\hline & & & (6) & & & & & & & & & & & & & \\
\hline & & $2 / 28$ & \#8 & & 29.3 & 28.4 & 28.8 & + & UD & NA & 39.1 & - & & & & - \\
\hline & & & (8) & & & & & & & & & & & & & \\
\hline & & $2 / 29$ & $\# 9$ & & & & & & & & & & 32.8 & 32.6 & 32.6 & \\
\hline & & & (9) & & & & & & & & & & & & & \\
\hline & & $3 / 02$ & $\# 11$ & & 31.8 & 31.6 & 32.0 & & UD & NA & NA & & 36.2 & NA & NA & - \\
\hline & & & $(11)$ & & & & & & & & & & & & & \\
\hline \#2 & No & $2 / 21$ & $\# 0$ & - & 20.7 & 21.3 & 21.5 & + & 36.3 & 37.1 & NA & - & & & & \\
\hline $30 / \mathrm{M}$ & picumina & & (0) & & & & & & & & & & & & & \\
\hline None & requirement) & $2 / 22$ & $\# 1$ & & & & & & & & & & 17.2 & 13.8 & 14.2 & + \\
\hline & / 30 & & (1) & & & & & & & & & & & & & \\
\hline & & $2 / 23$ & \#2 & & & & & & & & & & 17.3 & 17.8 & 18.0 & + \\
\hline & & & $(2)$ & & & & & & & & & & & & & \\
\hline & & $2 / 24$ & \#3 & & 25.1 & 26.1 & 26.0 & - & 24.3 & 17.9 & 26.0 & - & 27.4 & 29.6 & 28.9 & + \\
\hline & & & (3) & & & & & & & & & & & & & \\
\hline & & $2 / 25$ & $\# 4$ & & 23.9 & 24.9 & 24.9 & - & & & & & & & & \\
\hline & & & (4) & & & & & & & & & & & & & \\
\hline & & $2 / 26$ & \#5 & & 23.3 & 24.5 & 24.7 & + & 23.7 & 17.8 & 24.9 & - & & & & \\
\hline & & & (5) & & & & & & & & & & & & & \\
\hline & & $2 / 27$ & \#6 & & & & & & & & & & 20.3 & 23.9 & 21.9 & + \\
\hline & & & (6) & & & & & & & & & & & & & \\
\hline & & $2 / 28$ & \#7 & & 23.7 & 24.6 & 24.4 & - & 32.8 & 17.8 & 33.4 & - & 23.7 & 24.8 & 25.3 & - \\
\hline
\end{tabular}

cf HD: Hospital day, SD: Symptom onset to sampling day, BAL: Bronchoalveolar lavage, PCR: Polymerase chain reaction, IFA: Indirect immunofluorescence as severity index, Lop/r: lopinavir-ritonavir (Kaletra), HTN: Hypertension, DM: Diabetes mellitus, CAD: Coronary artery disease, UD: Undetermined, NA: Not applica 


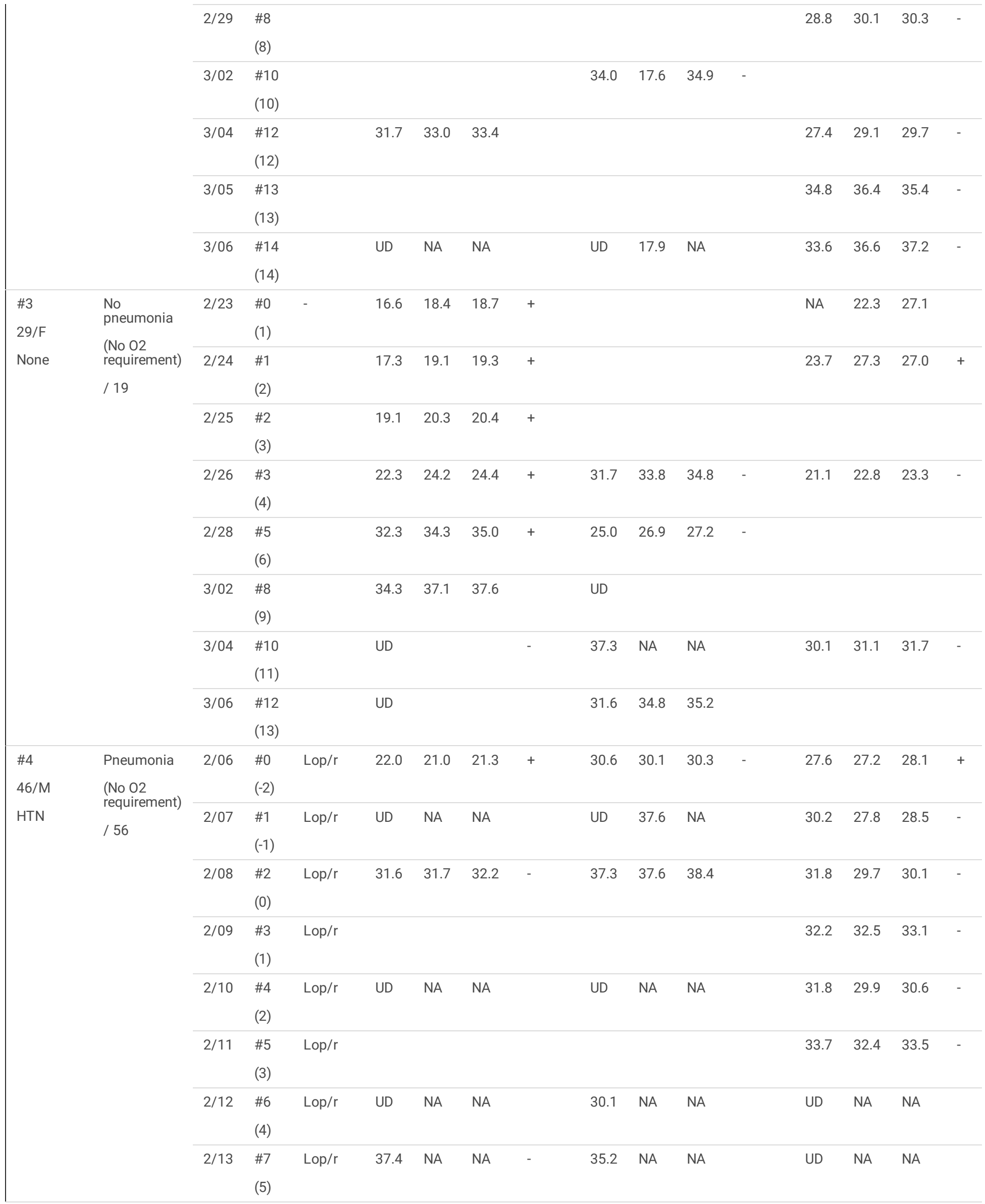




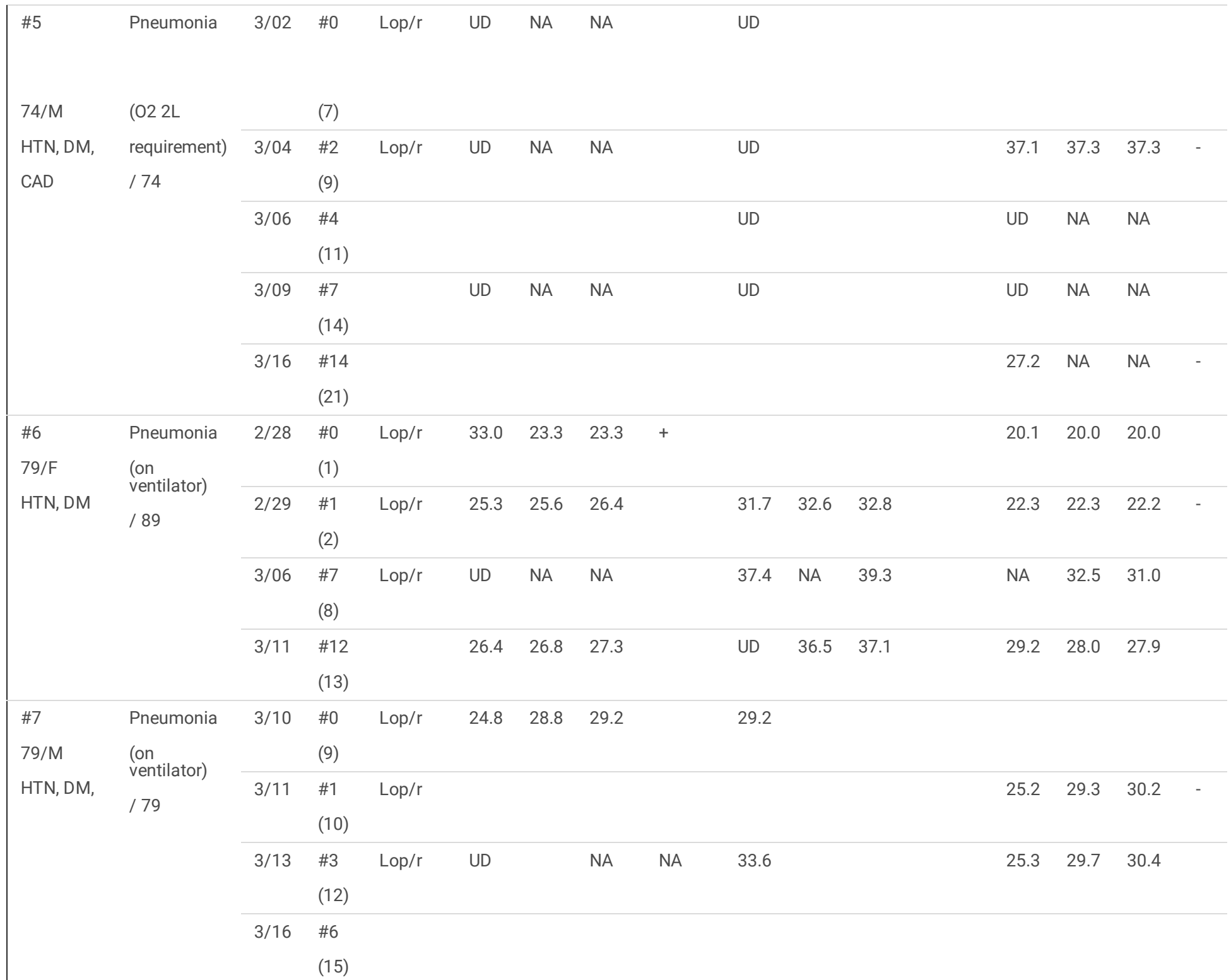

cf HD: Hospital day, SD: Symptom onset to sampling day, BAL: Bronchoalveolar lavage, PCR: Polymerase chain reaction, IFA: Indirect immunofluorescence as severity index, Lop/r: lopinavir-ritonavir (Kaletra), HTN: Hypertension, DM: Diabetes mellitus, CAD: Coronary artery disease, UD: Undetermined, NA: Not applica

Patient 4 was also identified through contact tracing. The patient presented with pneumonia confirmed by a computed tomography scan performed upon hospital admission. Nasopharyngeal and sputum specimen cultures were obtained on the same day, and viable SARS-CoV-2 was detected. The patient was administered $520 \mathrm{mg}$ lopinavir/125 mg ritonavir at 9 p.m. that day and tested negative in a culture test performed with sputum collected the next morning at 6 a.m. No virus was detected in any subsequent culture.

Patient 5 was administered lopinavir/ritonavir at another hospital 3 days before being transferred to Chosun University Hospital for oxygen therapy. No virus was detected in cultures performed during hospital admission.

Patient 6 was in critical condition and was intubated upon arrival at Chosun University Hospital. The nasopharyngeal culture was performed immediately to detect the virus. The patient was administered $520 \mathrm{mg}$ lopinavir/125 mg ritonavir twice on the first day, and the virus was detected neither in the sputum on the second day nor in the bronchoalveolar lavage (BAL) fluid cultures performed on the 12th day.

Patient 7 was administered lopinavir/ritonavir at another hospital 4 days before admission at Chosun University Hospital for acute respiratory disease syndrome and immediately placed on a mechanical ventilator. The virus was not detected in cultures upon admission or in BAL fluid culture performed on the third day after hospital admission (Table 1).

\section{Discussion}

False-negative PCR results may lead to inadequate isolation of infectious patients and consequently cause more infections. ${ }^{7}$ In contrast, false positives cause panic among healthcare professionals and waste human and material resources due to unnecessary isolation and testing of contacts. ${ }^{8}$

Page $7 / 11$ 
A Taiwanese study examining 2,500 close contacts of 100 COVID-19 patients reported that all 22 secondary cases had their first exposure to COVID-19 within 6 days of symptom onset in the index case, and none of the 850 contacts who were in contact thereafter were infected. ${ }^{6}$

A Chinese study reported that COVID-19 transmission may occur as early as 2.3 days before symptom onset and peak transmissibility is reached at 0.7 days before symptom onset. The transmissibility gradually decreases thereafter. ${ }^{9}$

Furthermore, since the infectious virus is rarely isolated from immunocompetent patients $7-10$ days after symptom onset, the CDC suggests discontinuing precautions and isolation after 10 days. ${ }^{10}$ However, studies have detected replication-competent viruses in respiratory or fecal specimens from patients in severe or critical conditions and immunocompromised COVID-19 patients for over 10 days after symptom onset. ${ }^{11,12}$

It has also been reported that asymptomatic or pre-symptomatic patients infected with SARS-CoV-2 can transmit the infection. ${ }^{13}$ In our study, the virus was detected in Patient 4 in the pre-symptomatic stage. The virus was detected in patients with mild symptoms or those not administered antiviral agents in the pre-symptomatic stage. The virus was detected for up to 8 days after symptom onset in patient 1.

In South Korea, all COVID-19 patients are isolated in negative pressure rooms or community treatment centers regardless of whether they are symptomatic or asymptomatic. ${ }^{14,15}$

When practicing test-based strategies, isolation is discontinued only if the patient tests negative in two real-time PCR tests performed at least $24 \mathrm{~h}$ apart. However, prolonged viral RNA detection does not indicate prolonged transmissibility. ${ }^{16}$

The reported Ct of viable SARS-CoV-2 detection varies widely from $<24$ to $<32 .{ }^{17,18}$ A study reported that the infectious virus was not detected when the viral RNA load was $<10^{6} .{ }^{16}$ However, previous studies did not consider the effects of antiviral agents. In this study, we observed that the virus was detected for up to 8 days after symptom onset or hospital admission in patients with mild symptoms and without lopinavir/ritonavir administration. However, no viable virus was detected in any of the specimens obtained from patients who were administered lopinavir/ritonavir.

An open-label randomized trial with patients hospitalized for COVID-19 reported that a 10-day course of lopinavir-ritonavir did not reduce 28-day mortality or mechanical ventilation requirements. ${ }^{19}$ Analysis of tissue distributions of antiviral drugs to reduce viral loads in COVID-19 treatment revealed that drugs with poor lung distributions, such as lopinavir/ritonavir, umifenovir, and remdesivir, were insufficient to inhibit viral replication. Based on the distribution profile, lopinavir/ritonavir might inhibit SARS-CoV-2 more efficiently in the gastrointestinal tract. ${ }^{20}$ Therefore, lopinavir/ritonavir administration may not be appropriate for therapeutic purposes.

Lopinavir/ritonavir showed significant inhibitory effects on SARS-CoV-2 in vitro at its usual plasma concentration. ${ }^{21}$ Lopinavir is a proteinase inhibitor that blocks a post-entry step in the Middle Eastern respiratory syndrome coronavirus replication cycle. ${ }^{22}$ This study suggests that respiratory specimens in patients who were administered lopinavir/ritonavir may contain the drug that inhibits SARS-CoV-2.

To our knowledge, no previous study has explored the possibility of using lopinavir/ritonavir to reduce infectious viral shedding or control infections. The results of this study suggest that immunocompetent patients administered lopinavir-ritonavir may discontinue infection control precautions within 10 days; this would lead to more flexible operation of hospital wards and shorten the isolation period of patients, thereby combating healthcare system overload by increasing the available number of intensive care unit beds.

Our study suggests that more research is needed on the effect and mechanism of action of lopinavir-ritonavir in COVID-19 transmissibility, along with research on the capacity of short-term lopinavir-ritonavir administration to induce viral resistance as well as any subsequent side effects of the treatment. It would be prudent to investigate the effect of other antiviral agents such as remdesivir on COVID-19 transmissibility.

A limitation of this study is that it was a single-institution study with a small sample size of seven patients. Only four patients who received treatment with lopinavir/ritonavir underwent culture testing.

To the best of our knowledge, this study is the first to report that SARS-CoV-2 may be continuously detected in patients with mild symptoms who are not administered antiviral agents for up to 8 days and that viable SARS-CoV-2 is not detected in immunocompetent COVID-19 patients after lopinavir/ritonavir administration. This study suggests that lopinavir/ritonavir may have some effect on the transmissibility of COVID-19; therefore, its use may provide a suitable option to reduce the isolation time of affected patients.

\section{Declarations}

\section{Ethics approval and consent to participate:}

The study was approved by the Institutional Review Board of Chosun University Hospital (CHOSUN 2020-04-003-002). Written informed consent was obtained from all participants of this study.

\section{Consent for publication:}

Not applicable

Availability of data and materials: 
The data set supporting the conclusion of article are included within the article.

\section{Competing interests:}

The authors declare that they have no competing interests.

\section{Funding:}

None

\section{Author contributions:}

DM Kim conceived and drafted the manuscript. JK Chung and JJ Seo contributed to the methodology and investigation. CM Kim and YM Lee contributed to the methodology and revised the manuscript. MS Bang and M Tariq contributed to the investigation and revised the manuscript. JW Seo, NR Yun, and DY Kim collected the data and performed the analysis. Y Kim and MJ Kim contributed to the investigation and revised the manuscript. $\mathrm{NH}$ Cho conceived and revised the manuscript. All authors have read and approved the final manuscript.

\section{Acknowledgments:}

None

\section{References}

1. Gorbalenya, A. E. et al. Severe acute respiratory syndrome-related coronavirus: The species and its viruses - a statement of the Coronavirus Study Group. bioRxiv (2020) doi:10.1101/2020.02.07.937862.

2. Ecdc. Suggested citation: European Centre for Disease Prevention and Control. Rapid increase of a SARS-CoV-2 variant with multiple spike protein mutations observed in the Rapid increase of a SARS-CoV-2 variant with multiple spike protein mutations observed in t. http://covid19-countryoverviews.ecdc.europa.eu/\#34_United_Kingdom (2020).

3. CDC. Disposition of Non-Hospitalized Patients with COVID-19. Centers for Disease Control and Prevention https://www.cdc.gov/coronavirus/2019ncov/hcp/disposition-in-home-patients.html\#previous (2020).

4. World Health Organization. Criteria for releasing COVID-19 patients from isolation. Sci. Br. 1-5 (2020).

5. Fontana Do, L. M., Holly Villamagna, A., Sikka, M. K. \& Mcgregor, J. C. Understanding viral shedding of severe acute respiratory coronavirus virus 2 (SARSCoV-2): Review of current literature. Infect. Control Hosp. Epidemiol. 1-10 (2020) doi:10.1017/ice.2020.1273.

6. Cheng, H.-Y. et al. Contact Tracing Assessment of COVID-19 Transmission Dynamics in Taiwan and Risk at Different Exposure Periods Before and After Symptom Onset. JAMA Intern Med 180, 1156-1163 (2020).

7. Woloshin, S., Patel, N. \& Kesselheim, A. S. False Negative Tests for SARS-CoV-2 Infection - Challenges and Implications. N. Engl. J. Med. 383, e38 (2020).

8. Jia, X., Xiao, L. \& Liu, Y. False negative RT-PCR and false positive antibody tests-Concern and solutions in the diagnosis of COVID-19. Journal of Infection (2020) doi:10.1016/j.jinf.2020.10.007.

9. He, X. et al. Temporal dynamics in viral shedding and transmissibility of COVID-19. Nat. Med. 26, 672-675 (2020).

10. Centers for Disease Control and Prevention. Duration of Isolation and Precautions for Adults with COVID-19_ CDC. Centers Dis. Control Prev. 2019, 1-7 (2020).

11. Avanzato, V. A. et al. Case Study: Prolonged Infectious SARS-CoV-2 Shedding from an Asymptomatic Immunocompromised Individual with Cancer. Cell 183, 1901-1912.e9 (2020).

12. Choi, B. et al. Persistence and Evolution of SARS-CoV-2 in an Immunocompromised Host. N. Engl. J. Med. 383, 2291-2293 (2020).

13. Bai, Y. et al. Presumed Asymptomatic Carrier Transmission of COVID-19. JAMA - Journal of the American Medical Association vol. $3231406-1407$ (2020).

14. Disease, C., Headquarters, C., Disaster, C. \& Headquarters, M. Response Guidelines To Prevent the Spread of COVID-19 At Public and Multi-purpose Facilities. (2020).

15. Kang, E. K. et al. Operating Protocols of a Community Treatment Center for Isolation of Patients with Coronavirus Disease, South Korea. Emerg. Infect. Dis. 26, 2329-2337 (2020).

16. Wölfel, R. et al. Virological assessment of hospitalized patients with COVID-2019. Nature 581, 465 (2020).

17. Basile, K. et al. Cell-based culture of SARS-CoV-2 informs infectivity and safe de-isolation assessments during COVID-19. medRxiv (2020) doi:10.1101/2020.07.14.20153981.

18. Bullard, J. et al. Clinical Infectious Diseases Predicting Infectious Severe Acute Respiratory Syndrome Coronavirus 2 From Diagnostic Samples. Clin. Infect. Dis. \& 71, 2663-2669 (2020).

19. Horby, P. W. et al. Lopinavir-ritonavir in patients admitted to hospital with COVID-19 (RECOVERY): a randomised, controlled, open-label, platform trial. Lancet 396, 1345-1352 (2020).

20. Sims, A. C. et al. Release of Severe Acute Respiratory Syndrome Coronavirus Nuclear Import Block Enhances Host Transcription in Human Lung Cells. J. Virol. 87, 3885-3902 (2013). 
21. Josset, L. et al. Cell host response to infection with novel human coronavirus EMC predicts potential antivirals and important differences with SARS coronavirus. MBio 4, (2013).

22. De Wilde, A.H. et al. Screening of an FDA-approved compound library identifies four small-molecule inhibitors of Middle East respiratory syndrome coronavirus replication in cell culture. Antimicrobial agents and chemotherapy 58, 4875-4884 (2014).

\section{Figures}
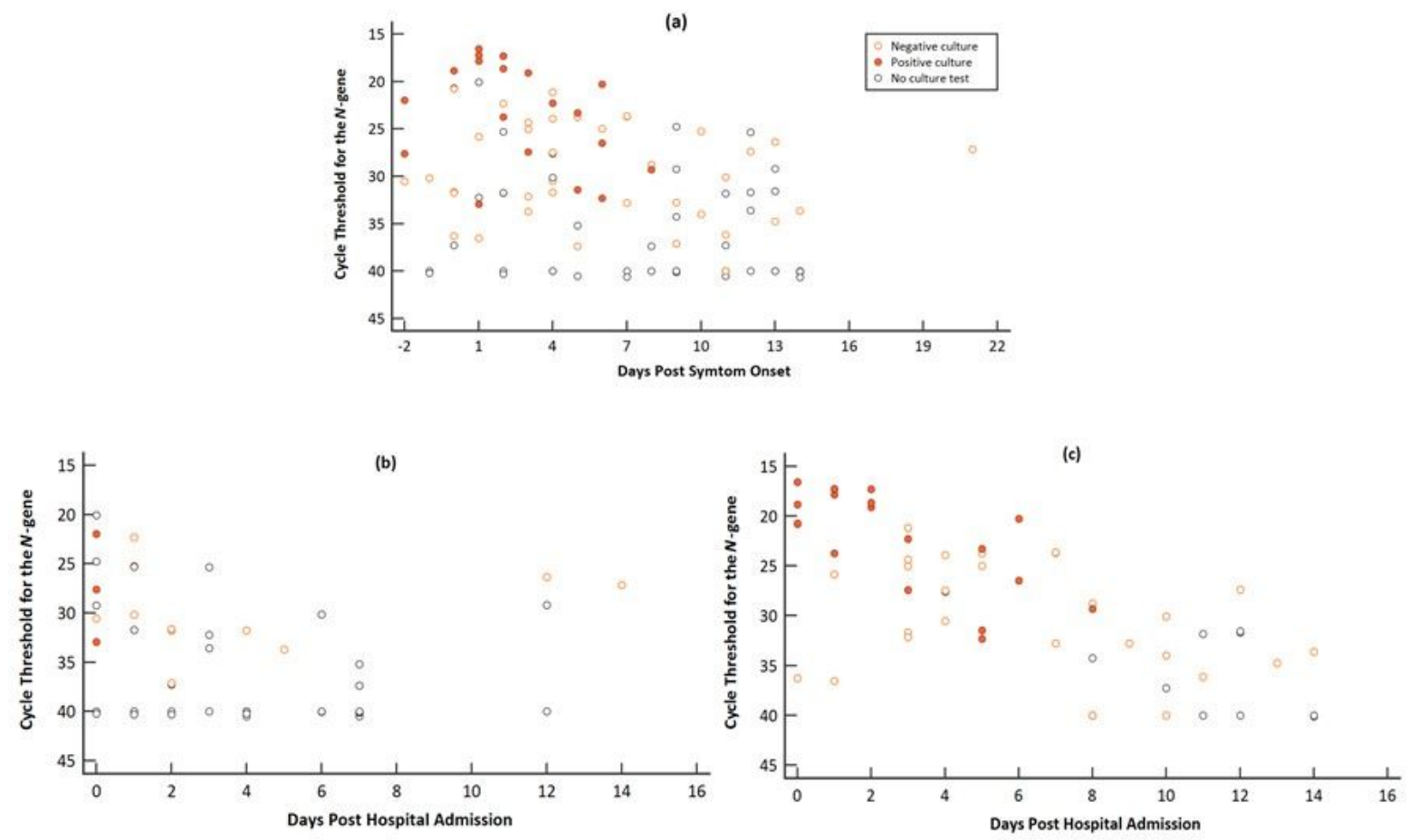

\section{Figure 1}

Timing of presence or absence of viable SARS-CoV-2 on Vero E6 cells and cycle threshold values of (a) all 104 serial respiratory samples obtained from seven patients diagnosed with COVID-19, (b) 47 serial respiratory samples obtained from four patients treated with lopinavir/ritonavir during their hospital stay, and (c) 57 serial respiratory samples obtained from three patients not treated with any antiviral agent during the complete course of their hospital stay.

(a)

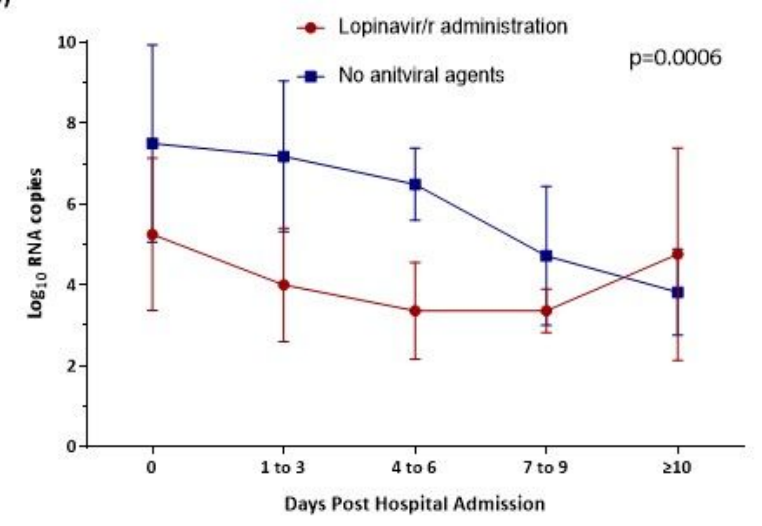

\begin{tabular}{ccccccc} 
Days post hospital admis sion & $\#$ & 0 & 1 to 3 & 4 to 6 & 7 to 9 & $\geq 10$ \\
\hline \multirow{3}{*}{ Lopinavir/r administration } & Mean & 5.25 & 4.00 & 3.36 & 3.36 & 4.76 \\
& SD & 1.89 & 1.40 & 1.20 & 0.55 & 2.62 \\
& $\mathrm{~N}$ & 7 & 10 & 5 & 6 & 2 \\
\hline \multirow{3}{*}{ No antiviral agents } & Mean & 7.50 & 7.18 & 6.49 & 4.72 & 3.82 \\
& SD & 2.43 & 1.86 & 0.89 & 1.72 & 1.06 \\
& $\mathrm{~N}$ & 4 & 8 & 9 & 6 & 10 \\
\hline
\end{tabular}

(b)

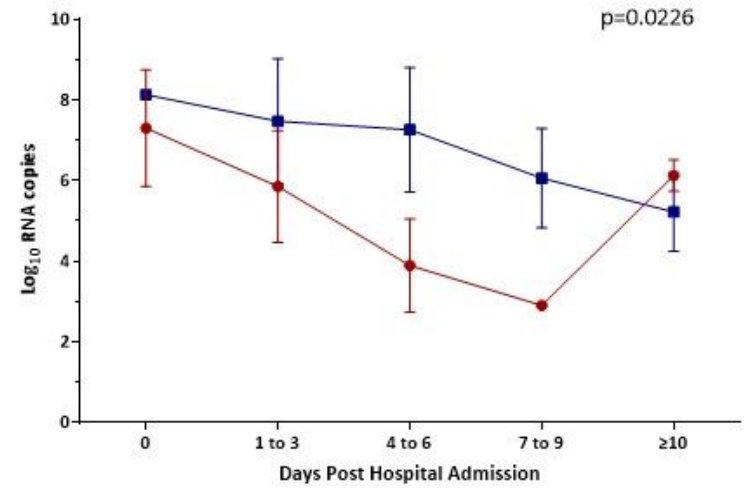

\begin{tabular}{ccccccc} 
Days post hospital admission & $\#$ & 0 & 1 to 3 & 4 to 6 & 7 to 9 & $\geq 10$ \\
\hline \multirow{3}{*}{ Lopinavir/r administration } & Mean & 7.30 & 5.85 & 3.89 & 2.90 & 6.12 \\
& SD & 1.45 & 1.39 & 1.16 & 0.00 & 0.39 \\
& $\mathrm{~N}$ & 2 & 7 & 4 & 2 & 2 \\
\hline \multirow{3}{*}{ No antiviral agents } & Mean & 8.13 & 7.47 & 7.26 & 6.06 & 5.22 \\
& SD & 0.00 & 1.55 & 1.54 & 1.24 & 0.98 \\
& $\mathrm{~N}$ & 1 & 7 & 3 & 3 & 5 \\
\hline
\end{tabular}

Figure 2

Comparison of viral RNA loads (log10 RNA copies, N-gene) at days post-hospital admission in (a) upper and (b) lower respiratory tract samples from patients treated with lopinavir/ritonavir or not treated with antiviral agents. SD; standard deviation and N; number of samples p-value is calculated by comparison of 
means (t-test) Upper respiratory tract includes oropharyngeal and nasopharyngeal swabs while lower respiratory tract includes sputum samples; Day 0 indicates day of hospital admission and day of antiviral administration

\section{Supplementary Files}

This is a list of supplementary files associated with this preprint. Click to download.

- Figures1.docx

- Figures2.docx 